\title{
Investigation on Insulating Properties of the Oil-Paper Transformer at its Different Position by Partial Discharging System
}

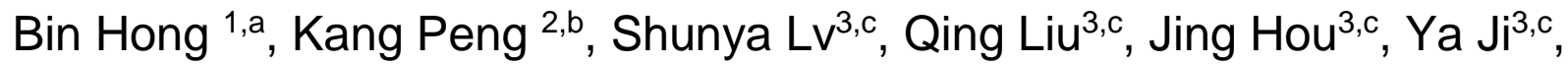 \\ Zhendong $\mathrm{Gao}^{3, \mathrm{c}}$, Jianye $\mathrm{Gao}^{3, \mathrm{c}}, \mathrm{Ju} \mathrm{Hu}^{3, \mathrm{c}}$ \\ ${ }^{1}$ Internal Combustion Engine Research Institute, Tianjin University, Tianjin 300072, China \\ ${ }^{2}$ Institute of Electronic Engineering, China Institute of Engineering Physics, Sichuan 621900, China \\ ${ }^{3}$ Tianbo Science \& Technology Co. Ltd., Tianjin 300072, China \\ atianbohb@126.com, btianbojy905@126.com, ‘630540402@qq.com
}

Keywords: insulating property, oil-paper transformer, partial discharging system, vacuum pouring technology.

\begin{abstract}
The oil-paper transformer is widely used as the common insulating device in the electrical industry. The transformer with the reserved primary coil and the secondary coils was filled with the transformer oil by the vacuum pouring process. Its insulating property was tested by the partial discharging (PD) system and the testing results are analyzed in detail. The experiment data reveals that the insulating properties of transformer at different positions are diverse due to the specific oil-paper structure. Obviously, the outer part of the transformer shows a much higher insulating property than its inner part. This confirms the residual air or defects are mostly concentrated in the inner part and air in the outer part is easier to be degassed by a viable vacuum pouring technology.
\end{abstract}

\section{Introduction}

Power transformer insulation is one of the most important pieces of equipment in electrical systems, which is critical and expensive in the field of transmission and distribution of electric energy [1,2]. It has the insulation systems made in cellulose paper and mineral oil [3], which are used in the electric power transformer mainly to transfer heat [4].

Characteristically, the insulating oil used in electric transformers consists of complex blends of more than 3000 hydrocarbons and they are essentially highly branched aliphatics, naphthenic or paraffinic crudes. With the long term suffering to the electrical, mechanical, thermal and environment time variable stresses, the paper and oil decomposition takes place and generates gases that dissolve in the oil and decrease its dielectric strength [5]. Thus, the air effect as the most dangerous factor causes failures of power transformers during which a violent release of water vapor from cellulose insulation takes place after exceeding a critical temperature and a critical moisture content [6]. Nowadays, many transformers are located in populated areas and shopping centers, and these changes in its physio-chemical characteristics make it unfit for insulating purpose in an electric transformer [7,8,9], which may continually cause not only catastrophic damage to various equipments but interruption of electricity supply. Therefore, the evaluation of the insulating property for the power transformer is quite essential which has attracted great attentions in recent years. 
Partial discharge (PD), if occurring in oil-immersed power transformer, is considered to damage insulators, and the PD measurement is conducted for an actual transformer in the long-duration AC withstand voltage test [10,11]. Typically, a transformer incorporates coils of conducting wire wrapped around a core and covered with a paper-based insulator [12]. Due to this specific structure, the residual air and the generated air inside the insulation are always difficult to be degassed which will strongly affect its insulating property and rapidly decrease its life-time. Herein, a power transformer was obtained by a vacuum pouring process with insulating paper immersed transformer oil. It reserved a primary coil and three secondary coils of conducting wire that wrapped around a core and covered with a paper based insulator. The primary coil and secondary coils of the transformer insulation which is the decisive factor to its continued operation [13], were respectively introduced to the PD measurement as the testing electrodes in order to evaluate the insulating properties and the distribution of the air defects inside the transformer. The testing data was analyzed and the insulating properties of transformer at different positions are compared. Furthermore, the distribution law of the air defects inside the transformer insulation diverse is deeply discussed according to the testing results.

\section{Experiments}

\section{Preparation of the oil-paper transformer insulation}

As shown in Fig.1, the tested transformer insulation was obtained by a vacuum pouring technology with the insulating papers immersed insulating oil. The coils of conducting wire that wrapped around a core were reserved and covered with a paper based insulator. Among these coils, the primary coil is cling to the core and the secondary coils containing three coils are orderly distributed in different paper layers which here are named as the secondary-1st coil, the secondary-2nd coil and the secondary-3rd coil, respectively.

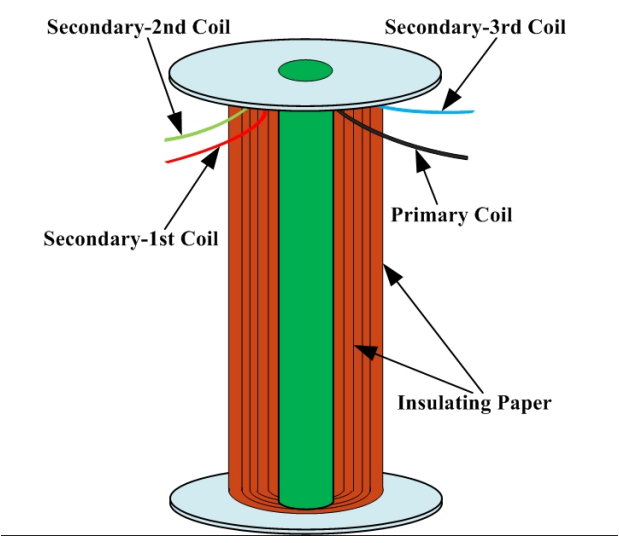

Fig.1 Structure Diagram of the tested transformer insulation

The pouring process of the transformer insulation is displayed in Fig.2: The assembled transformer was firstly degassed in the vacuum chamer and the inside pressure was controlled at about $500 \mathrm{~Pa}$. Then turn on the switch and insulating oil was pouring into the transformer. With the stable vacuum pressure, the transformer oil can partly soak into the spaces between the adjacent insulating papers. 


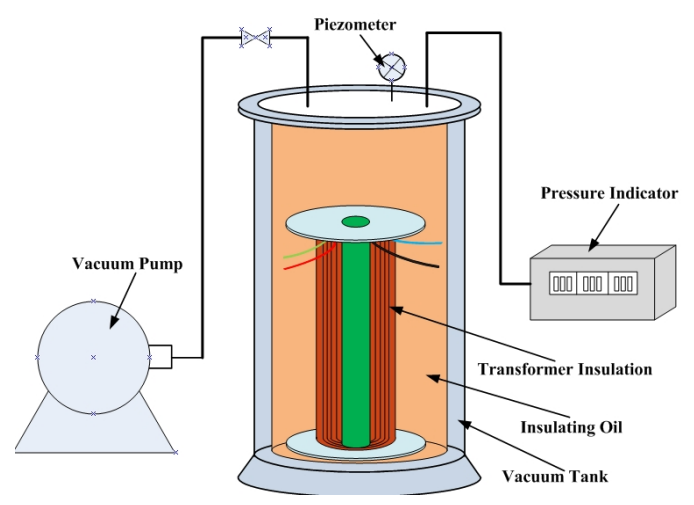

Fig.2 The vacuum pouring process of the transformer insulation filled with insulating oil

\section{Partial discharging process of the transformer insulation}

In order to test the insulating properties and analysis the distribution of the air defects inside the transformer, the reserved coils were introduced into the testing system as the two voltage electrodes. The testing voltage was increased gradually and the initial discharging voltage was recorded when the partial discharging occurs during the resting process. Generally speaking, the testing results are seriously influenced by the adopted testing method. Therefore, the combination of impulse current and oscilloscope is introduced into the testing system for the PD analysis of the transformer insulation.

\section{Results and discussion}

\section{Analysis of PD testing for the transformer}

The PD testing result of the transformer insulation is exhibited by Fig.3 which the initial discharging can be obviously found when the loaded AC voltage is increased to $3.1 \mathrm{KV}$. As shown in Fig.3 (a) and (b), the discharge capacity of the transformer is about $12 \mathrm{mV}$ and it discharges frequently. It indicates the initial discharging of the transformer insulation is only $3.1 \mathrm{KV}$ and lots of air bubbles are formed inside the insulation. In other words, the transformer oil was soak into the insulation partly and the inside air did not be exhausted.

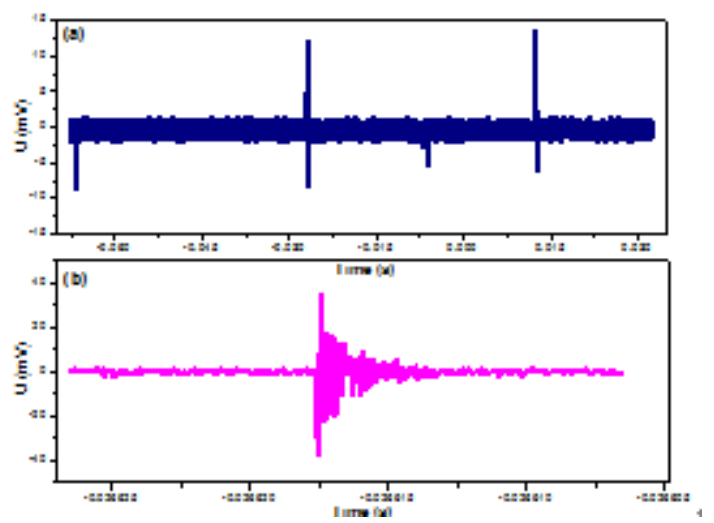

Fig.3 PD results of the transformer with the AC voltage at $3.1 \mathrm{KV}$

\footnotetext{
Insulating analysis of PD testing results between the primary coil and the secondary-1st coil

The insulating property of the spaces between the primary coil and its secondary-1st coil was tested. As seen in Fig.4 (a) and (b), the insulation discharged slightly when the AC voltage increased to $3.6 \mathrm{KV}$ with a discharging capacity of $15 \mathrm{mV}$. Moreover, some discharging waveforms
} 
are asymmetric with lots of discharging glitches but some are presented as the typical symmetric damped oscillation wave. As the loaded voltage is continuously increased to $4.2 \mathrm{KV}$, the partial discharging phenomenon of the transformer can be observed distinctly.
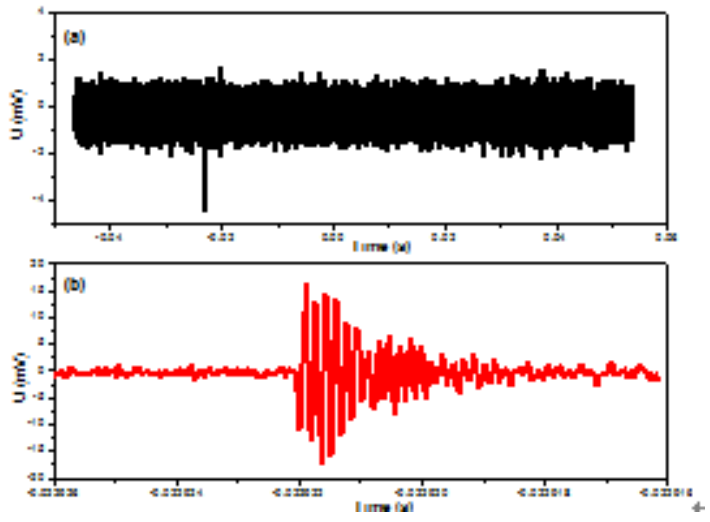

Fig.4 The PD results of the spaces between the primary coil and the secondary-1st coil with the AC voltage at $3.6 \mathrm{KV}$

Insulating analysis of PD testing results between the secondary-1st coil and the secondary-2nd coil

The PD results of the spaces between the transformer's secondary-1st coil and its secondary-2nd coil is displayed in Fig. 5 by the relevant coils are introduced into the testing system. During the PD testing process, few partial discharging phenomenon are observed which are aggravated as the loaded voltage is raised up to $4.9 \mathrm{KV}$. As presented in Fig.5, the transformer discharges strongly with a large discharging capacity of $50 \mathrm{mV}$. Compared with the PD testing results exhibited in Fig.4, the insulating property of the spaces between the sencondary-1st coil and the secondary- $2^{\text {nd }}$ coil is much better as the initial discharging voltage is increased from $3.6 \mathrm{KV}$ to 4.9 KV. It may be inferred that the transformer oil can be easier soaked into the outer spaces than inner spaces of the oil-paper structure.

Insulating analysis of PD testing results between the secondary-2nd coil and the secondary-3rd coil

Fig.6 shows the PD results of the spaces between the transformer's secondary-2nd coil and its secondary-3rd coil when the loaded voltage is increased to $5.5 \mathrm{KV}$. Distinctly, its initial discharging voltage is much higher compared with those presented in Fig. 4 and Fig.5. In this
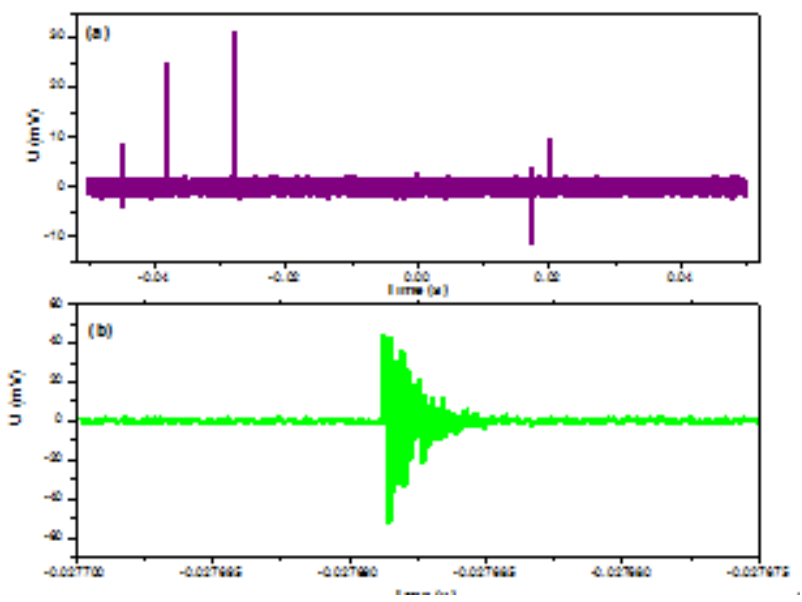

Fig.5 The PD results of the spaces between the secondary-1st coil and the secondary-2nd coil with 
the $\mathrm{AC}$ voltage at $4.9 \mathrm{KV}$

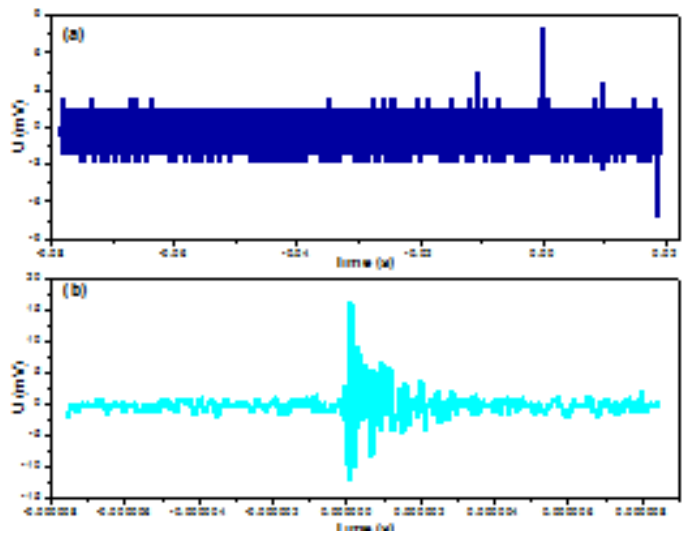

Fig.6 The PD results of the spaces between the secondary-2nd coil and the secondary-3rd coil with the AC voltage at $5.5 \mathrm{KV}$

occasion, the transformer discharges frequently but with lower discharging capacity. During the testing process, the symmetric damped oscillation wave is difficult to be captured and most discharging waves are emerged with lots of low discharging glitches. This result reconfirms that the soaking process of the transformer oil is much easier into the outer spaces than inner spaces of the oil-paper structure which is according with the above analysis.

According to the above analysis, the PD results of the transformer's different positions are summarized in table.1 which can clearly expressing the diverse insulating distribution of its insulating property. Due to the residual air inside the transformer, it discharged when the loaded AC voltage is only raised up to $3.1 \mathrm{KV}$ which the primary coil and the secondary-3rd coil are introduced into the PD testing system. Furthermore, the initial discharging voltages of the transformer's different positions are rapidly increased when the transformer is tested outward gradually. This can be induced by the residual air which means that there are more air remained in the inner part than the outer part. It can strongly manifest that the outer part of the transformer shows a better insulating property compared with the inner part. In other words, it reveals distinctly that the transformer oil is much easier to be soaked into the outer part than the inner part of the oil-paper insulating transformer.

Table.1 PD testing results of the transformer at different positions

\begin{tabular}{|c|c|c|}
\hline Testing Positions & $\begin{array}{c}\text { Initial } \\
\text { Discharging } \\
\text { Voltage }\end{array}$ & $\begin{array}{c}\text { Insulating } \\
\text { Property }\end{array}$ \\
\hline $\begin{array}{c}\text { Transformer insulation } \\
\text { (Spaces between the primary coil and the secondary-3rd coil) }\end{array}$ & $3.1[\mathrm{KV}]$ & + \\
\hline Spaces between the primary coil and the secondary-1st coil & $3.6[\mathrm{KV}]$ & ++ \\
\hline Spaces between the secondary-1st coil and the secondary-2nd coil & $4.9[\mathrm{KV}]$ & +++ \\
\hline Spaces between the secondary-2nd coil and the secondary-3rd coil & $5.5[\mathrm{KV}]$ & ++++ \\
\hline
\end{tabular}

\section{Conclusions}

The insulating property is the most important target for the evaluation of the transformer insulation. Due to the specific structure of the oil-immersed-paper transformer, the insulating properties are diverse to its different positions. According to the PD results, the outer part of the 
transformer shows a much higher insulating property than its inner part which means that the air defects are mostly distributed in the inner part and air in the outer part is easier to be degassed with the stable vacuum pouring process. This study is essential to the further research for the transformer insulation and it can provide effective experimental foundation for the insulating device.

\section{References}

[1] Dashti H, Sanaye-Pasand M. Power Transformer Protection Using a Multiregion Adaptive Differential Relay [J]. IEEE Transactions on Power Delivery, 2014, 29(2): 777-785.

[2] Liao R, Guo C, Wang $\mathrm{K}$ et al. Investigation on thermal aging characteristics of vegetable oil-paper insulation with flowing dry air [J]. IEEE Transactions on Dielectrics \& Electrical Insulation, 2013, 20(5): 1649-1658.

[3] Dumitran L M, Setnescu R, Notingher P V et al. Method for lifetime estimation of power transformer mineral oil [J]. Fuel, 2014, 117(1): 756-762.

[4] Jada A, Chaou A A, Bertrand Y et al. Adsorption and surface properties of silica with transformer insulating oils [J]. Fuel, 2002, 81(9): 1227-1232.

[5] Bakar N A, Abu-Siada A. A new method to detect dissolved gases in transformer oil using NIR-IR spectroscopy [J]. IEEE Transactions on Dielectrics \& Electrical Insulation, 2017, 24(1): 409-419.

[6] P. Przybylek, H. Moranda, K. Walczak, H. Moscicka-Grzesiak. Can the Bubble Effect Occur in an Oil Impregnated Paper Bushing? [J]. IEEE Transactions on Dielectrics and Electrical Insulation, 2012, 19 (6), 1879-1883.

[7] Kaanagbara L, Inyang H I, Wu J et al. Aromatic and aliphatic hydrocarbon balance in electric transformer oils [J]. Fuel, 2010, 89(10): 3114-3118.

[8] Lobeiras A, Sabau J. Particle counting of insoluble decay products in mineral insulating oils. Symp of ASTM; 2000.

[9] Yadav S P R, Saravanan C G, Vallinayagam R et al. Fuel and engine characterization study of catalytically cracked waste transformer oil [J]. Energy Conversion \& Management, 2015, 96: 490-498.

[10] Okabe S, Ueta G, Wada H. Partial Discharge Signal Propagation Characteristics inside the Winding of Oil-immersed Power Transformer Using the Three-Winding Transformer Model in Air [J]. IEEE Transactions on Dielectrics and Electrical Insulation, 2011, 18 (6): 2024-2031.

[11] Liao R J, Yang L J, Li J et al. Aging condition assessment of transformer oil-paper insulation model based on partial discharge analysis [J]. IEEE Transactions on Dielectrics \& Electrical Insulation, 2011, 18(1): 303-311.

[12] Bosworth T, Setford S, Heywood R et al. Pulsed amperometric detection of furan compounds in transformer oil [J]. Analytica Chimica Acta, 2001, 450(1-2): 253-261.

[13] Lelekakis N, Martin D, Wijaya J. Ageing rate of paper insulation used in power transformers Part 2: Oil/paper system with medium and high oxygen concentration [J]. IEEE Transactions on Dielectrics \& Electrical Insulation, 2013, 19(6): 2009-2018. 